\title{
Core-shell structures in single flexible-semiflexible block copolymers: Finding the free energy minimum for the folding transition
}

\section{AUTHOR(S):}

Yoshinaga, Natsuhiko; Yoshikawa, Kenichi

\section{CITATION:}

Yoshinaga, Natsuhiko ... [et al]. Core-shell structures in single flexible-semiflexible block copolymers: Finding the free energy minimum for the folding transition. Journal of Chemical Physics 2007, 127(4): 074904.

\section{ISSUE DATE:}

2007-07-28

URL:

http://hdl.handle.net/2433/45689

\section{RIGHT:}

Copyright (2007) American Institute of Physics. This article may be downloaded for personal use only. Any other use requires prior permission of the author and the American Institute of Physics. 


\title{
Core-shell structures in single flexible-semiflexible block copolymers: Finding the free energy minimum for the folding transition
}

\author{
Natsuhiko Yoshinaga \\ Department of Physics, Graduate School of Sciences, The University of Tokyo, Tokyo 113-0033, Japan \\ Kenichi Yoshikawa \\ Department of Physics, Graduate School of Sciences, Kyoto University, Kyoto 606-8502, Japan
}

(Received 1 May 2007; accepted 8 June 2007; published online 24 July 2007)

\begin{abstract}
We investigate the folding transition of a single diblock copolymer consisting of a semiflexible and a flexible block. We obtain a Saturn-shaped core-shell conformation in the folded state, in which the flexible block forms a core and the semiflexible block wraps around it. We demonstrate two distinctive features of the core-shell structures: (i) The kinetics of the folding transition in the copolymer are significantly more efficient than those of a semiflexible homopolymer. (ii) The core-shell structure does not depend on the transition pathway. (C) 2007 American Institute of Physics. [DOI: 10.1063/1.2753838]
\end{abstract}

\section{INTRODUCTION}

Polymer-based nanostructures have been extensively studied due to their importance in industrial applications, particularly in nanodevices and nanomachines. ${ }^{1-3}$ They are also of importance in living cells; DNA and protein molecules have nano-ordered structures, which show a close relationship with their biological functions. Macromolecules in biological systems typically undergo conformational transitions. In polymer physics, this has been discussed as the coil-globule transition, ${ }^{4}$ in which a flexible swollen homopolymer collapses into a spherical globular conformation. The globule state is liquidlike and disordered, whereas a lot of macromolecules, particularly biomacromolecules, have ordered, folded structures. For this reason, there has recently been much attention to semiflexible homopolymers, which show bending rigidity along the chain, and, as a result, have rodlike properties, although their contour lengths are long enough to exhibit overall fluctuations. A semiflexible homopolymer has been extensively studied as a model for DNA molecules. ${ }^{5-7}$ In these works, nano-ordered structures, such as toroids, cylinders, and rackets, were investigated experimentally, theoretically, and computationally. ${ }^{8}$ Due to these extensive studies, it was found that the formation of nano-ordered conformations in DNA is well reproduced in semiflexible polymers with homogeneous bending rigidity. In living systems, however, biopolymers such as proteins are, in general, heteropolymers with complicated sequences of amino acids. It is often mentioned that sequences within proteins are relevant for their conformations in the folded states. Therefore, most studies heretofore conducted have dealt with sequences in heteropolymers which lead to diversity in conformations. Nevertheless, our understanding of role of heterogeneity in bending rigidity is still primitive. In this article, we propose a minimum model to extend the concept of semiflexible polymers toward heteropolymers. A single block copolymer is the simplest extension of a single homopolymer in the direction of single heteropolymers such as proteins. To this end, we concentrate on a simple model: Diblock copolymers in which two blocks possess different levels of flexibility.

Assemblies of rod-coil copolymers have been first discussed in the context of polymer blends; ${ }^{9,10}$ they have recently been investigated in the context of polymer solutions, ${ }^{11}$ with regard to their applications in the field of nanocapsules. ${ }^{12}$ However, less attention has been paid to the properties of single rod-coil copolymers. In computer simulations, several novel structures were found by Cooke and Williams for multiblock rod-coil copolymers, which they refer to as semiflexible copolymers. The static properties of these species were studied. ${ }^{13}$ In the present study, we focus instead on the kinetics of the process of folding into ordered phases in single rod-coil diblock copolymers.

The kinetics and pathways of conformational transitions are relevant since it is well known that in experiments the results strongly depend on the method of preparation. For example, the authors demonstrated that the final structure in the folding transition of DNA depends on whether condensing agents or monovalent salts are added first. ${ }^{14}$ This result suggests that we must discuss not only static stability but also kinetics pathways for the folding transition. Along these lines, we have investigated that the dependence of folded structures of semiflexible polymers depend on kinetic pathways. ${ }^{8,15}$ Once a chain falls into a metastable state, it cannot escape to the most stable state under thermal fluctuations. For semiflexible polymers, even when the toroidal conformation is the most favorable, a small hairpinlike conformation in the early stage of the folding transition results in the formation of a cylindrical structure as a stable state. Due to a high free energy barrier, the cylinder does not make the transition to a toroid over a practical time scale. Our interest is in the kinetics of formation of specific nanoordered structures, and particularly on the pathways to folded states. We demonstrate that our single flexible-semiflexible copolymer avoids local free energy minima and forms a core-shell structure. 


\section{SIMULATIONS}

We carried out Langevin dynamics simulations for a single rod-coil block copolymer. A rod-coil copolymer consists of a flexible and a semiflexible block. Although rigid rods with infinitely large bending elasticity are often used for the rod block, in order to allow more general discussions, we used a semiflexible polymer with bending rigidity. We adapted a bead-spring model with the following potentials:

$$
\begin{aligned}
& V_{\text {beads }}=\frac{k}{2} \sum_{i}\left(\left|\mathbf{r}_{i+1}-\mathbf{r}_{i}\right|-a\right)^{2}, \\
& V_{\text {bend }}=\sum_{\alpha} \kappa_{\alpha} \sum_{i \in \alpha}\left(1-\cos \theta_{i}\right), \\
& V_{\mathrm{LJ}}=4 \sum_{\alpha, \beta} \epsilon_{\alpha \beta} \sum_{i \in \alpha, j \in \beta}\left[\left(\frac{a}{\left|\mathbf{r}_{i}-\mathbf{r}_{j}\right|}\right)^{12}-\left(\frac{a}{\left|\mathbf{r}_{i}-\mathbf{r}_{j}\right|}\right)^{6}\right],
\end{aligned}
$$

where $\mathbf{r}_{i}$ is the coordinate of the $i$ th monomer and $\theta_{i}$ is the angle between adjacent bond vectors. The subscripts $\alpha$ and $\beta$ denote $s$ (semiflexible) or $f$ (flexible) monomers. The monomer size $a$ was chosen as the unit length, and $k_{B} T$ as the unit energy. The excluded volume and short-ranged attractive interactions between monomers are included with the LennardJones potential with the coupling constant $\epsilon_{\alpha \beta}$, which determines the strength of the attractive interaction between monomers in the $\alpha$ and $\beta$ states. We set the spring constant to be $k=400$. The bending elasticity for a flexible block was chosen to be $\kappa_{f}=0$. We note that the persistence length of a semiflexible block can be written as $l_{p} T=\kappa_{s} a$. The length of each block was chosen as $N_{s}=N_{f}=128$.

The equation of motion is written as

$$
m \frac{d^{2} \mathbf{r}_{i}}{d t^{2}}=-\gamma \frac{d \mathbf{r}_{i}}{d t}-\frac{\partial V}{\partial \mathbf{r}_{i}}+\boldsymbol{\xi}_{i}
$$

where $m$ and $\gamma$ are the mass and friction constant of monomeric units, respectively. The unit time scale is $\tau=\gamma a^{2} / k_{B} T$. We set the time step as $0.01 \tau$, and use $m=1.0$ and $\gamma=1.0$. With these parameters, the relaxation time of the momentum of a monomer is sufficiently fast compared to the time scale of interest. Gaussian white noise $\xi_{i}$ satisfies a fluctuationdissipation relation,

$$
\left\langle\boldsymbol{\xi}_{i}(t) \cdot \boldsymbol{\xi}_{j}\left(t^{\prime}\right)\right\rangle=6 \gamma k_{B} T \delta_{i j} \delta\left(t-t^{\prime}\right) .
$$

\section{CORE-SHELL STRUCTURE}

First, we consider the system in nonselective solvents, i.e., $\epsilon_{s s}=\epsilon_{s f}=\epsilon_{f f}=\epsilon$. Figure 1 shows typical conformations of folded single rod-coil block copolymers. At sufficiently large $\epsilon$, a rod-coil copolymer in the elongated state undergoes a transition to the folded state. For small value of $\kappa_{s}$, the collapse is disordered, while a Saturn-shaped conformation is obtained when $\kappa_{s}$ is large. In this state, the flexible block at the core is surrounded by the semiflexible block [Fig. 1(II)]. Intuitively, our core-shell structure is a composite of a globule and toroid. This is a typical characteristic of block copolymers: The folded state of this block copolymer incorporates the characters of both flexible and semiflexible
(A)

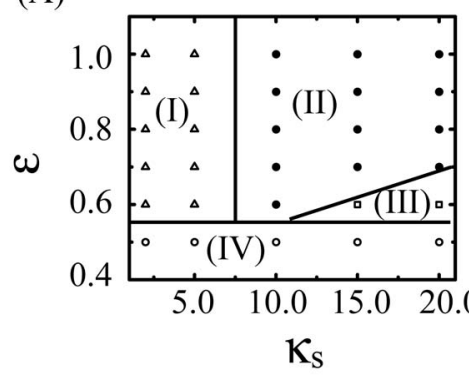

(I)

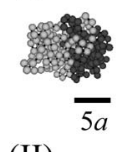

(III)

(II)
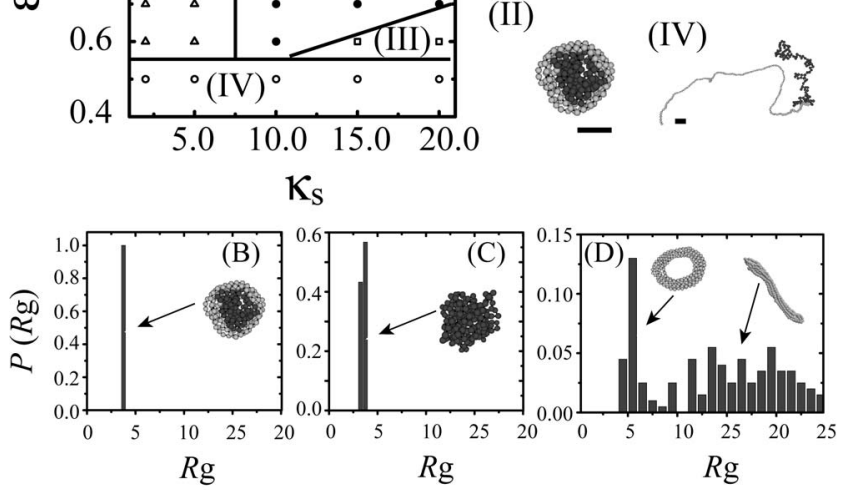

FIG. 1. Phase diagram of single folded rod-coil copolymers at various $\epsilon$ and $\kappa_{s}(\mathrm{~A})$, and illustrations of the conformations the polymer in each phase: (I) disordered collapse, (II) Saturn-shaped phase separation, (III) tadpole, and (IV) swollen. The probability distribution of the gyration radius of a rod-coil copolymer (B) and a flexible (C) and semiflexible (D) homopolymer in the collapsed state are also shown. In the figures, the monomer in the flexible block is shown in light gray, while the monomer in the semiflexible block is shown in dark gray. A semiflexible homopolymer has two stable conformations, toroidal and cylindrical.

polymers. This conformation is observed over a wide range of parameters, while at $\kappa_{s} \gg 0$ and $\epsilon \ll 1$, the semiflexible block is unable to fold, resulting in a tap-pole conformation with a long lifetime [Fig. 1(III)].

The core-shell structure is robust against the change of the length of blocks. The flexible block forms spherical shape, and thus, the size $R_{f}$ is proportional to

$$
R_{f} \sim N_{f}^{1 / 3} .
$$

Since the exponent is much smaller than 1 , the size is insensitive to the length. Furthermore, the size of the semiflexible block, $R_{s}$, is

$$
R_{s} \sim N_{s}^{1 / 5},
$$

which is also insensitive to the length of the block. In fact, both $N_{f}=64$ and $N_{f}=256$ blocks reproduce the core-shell structure in simulations (data not shown).

The final conformation of the rod-coil copolymer is the uniquely determined core-shell structure, in which the semiflexible block can only assume a toroidal conformation. In contrast, a semiflexible homopolymer possesses metastable conformations, such as a cylinder [Fig. 1(d)]. The toroidal and the cylindrical conformations are both stable, and, over the practical time scale, the distribution does not change since a transition between two states does not occur. We will come back to this point later.

We show in Fig. 2 the kinetics of the formation for a Saturn-shaped structure, where we suddenly increase $\epsilon$ at $t$ $=0$. The flexible block collapses at an early stage, and the semiflexible block then gradually wraps around it. This is in contrast to the situation for flexible and semiflexible homopolymers: flexible polymers exhibit spinodal decomposition in the folding process, ${ }^{16}$ whereas semiflexible polymers 


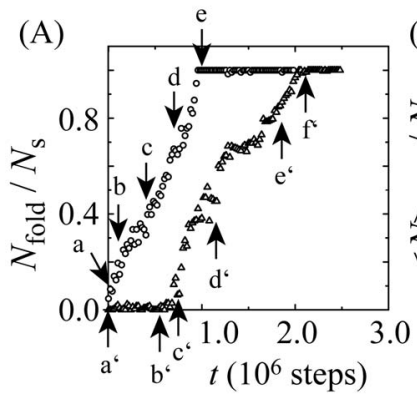

(B)
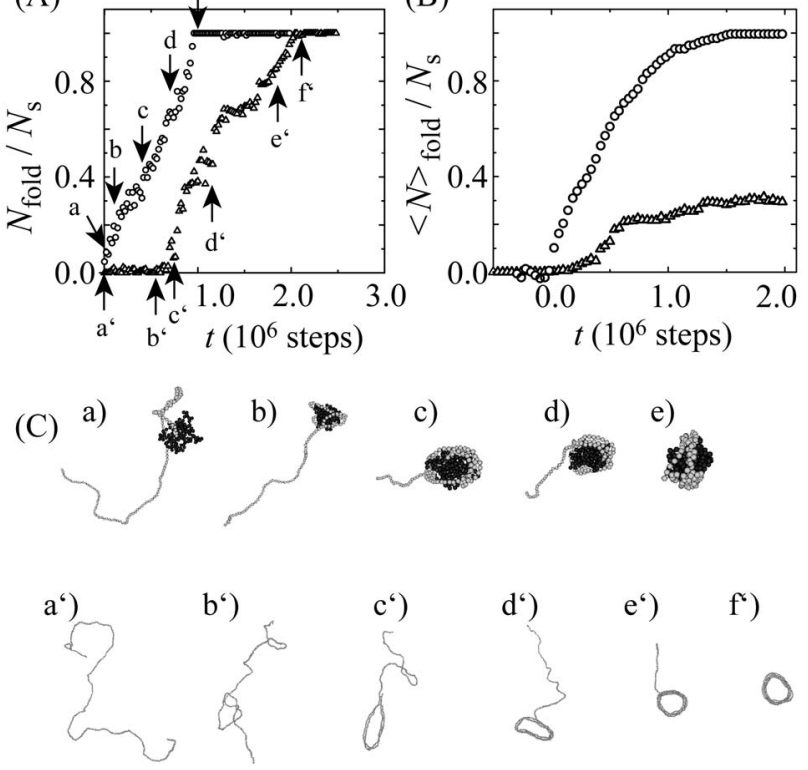

FIG. 2. Time evolution of the number of collapsed monomers in the semiflexible block of a rod-coil copolymer $(\bigcirc)$ and a semiflexible homopolymer $(\triangle)$. Each polymer has $N=256$ monomers, and a bending elasticity $\kappa_{s}=15$ for the semiflexible parts. We change the attractive interaction at $t=0$ to $\epsilon=0.8 . N_{\text {fold }}$ shows the number of monomers in the folded state. The bare data are shown in (A), while the mean evolution over 30 runs is shown in (B). Snapshots of the polymer conformation during the transition for a rodcoil copolymer $[(a)-(f)]$ and a semiflexible homopolymer $\left[\left(a^{\prime}\right)-\left(f^{\prime}\right)\right]$ are shown in $(\mathrm{C})$.

undergo a nucleation and coarsening process. ${ }^{5}$ Due to a stochastic feature of nucleation, there is a long lag time before the folding transition in a semiflexible polymer takes place. These observations indicate that a flexible polymer collapses much more quickly than a semiflexible polymer. Figure 2 shows the time evolution in the collapse ratio. As we can see, rod-coil copolymers undergo folding more quickly than semiflexible homopolymers. While at $t \sim 2 \times 10^{6}$, a rod-coil copolymer undergoes complete folding on average, only $30 \%$ of the monomers in a semiflexible homopolymer exhibit a collapsed state. The folding of semiflexible homopolymers is subject to a long lag time originating from the nucleation process, while rod-coil copolymers start to fold without such a process due to the existence of the flexibleblock core. As a result, the folding transition proceeds quickly.

These results are confirmed by the consideration of the time scale of folding in flexible and semiflexible polymers. ${ }^{17}$ The initial nucleation time depends exponentially on the persistence length of the polymer. This leads to a clear separation of the time scale between the flexible and semiflexible blocks. Thus, the folding kinetics of a rod-coil copolymer can be expected to be a two-step process: folding of the flexible block, and coarsening due to adsorption of the semiflexible block onto the globule of the flexible block.

\section{PATHWAYS}

Next, we move our focus to pathways to the folded state. Although in the above case all the parameters were quenched simultaneously, this is not always the case. For this reason,
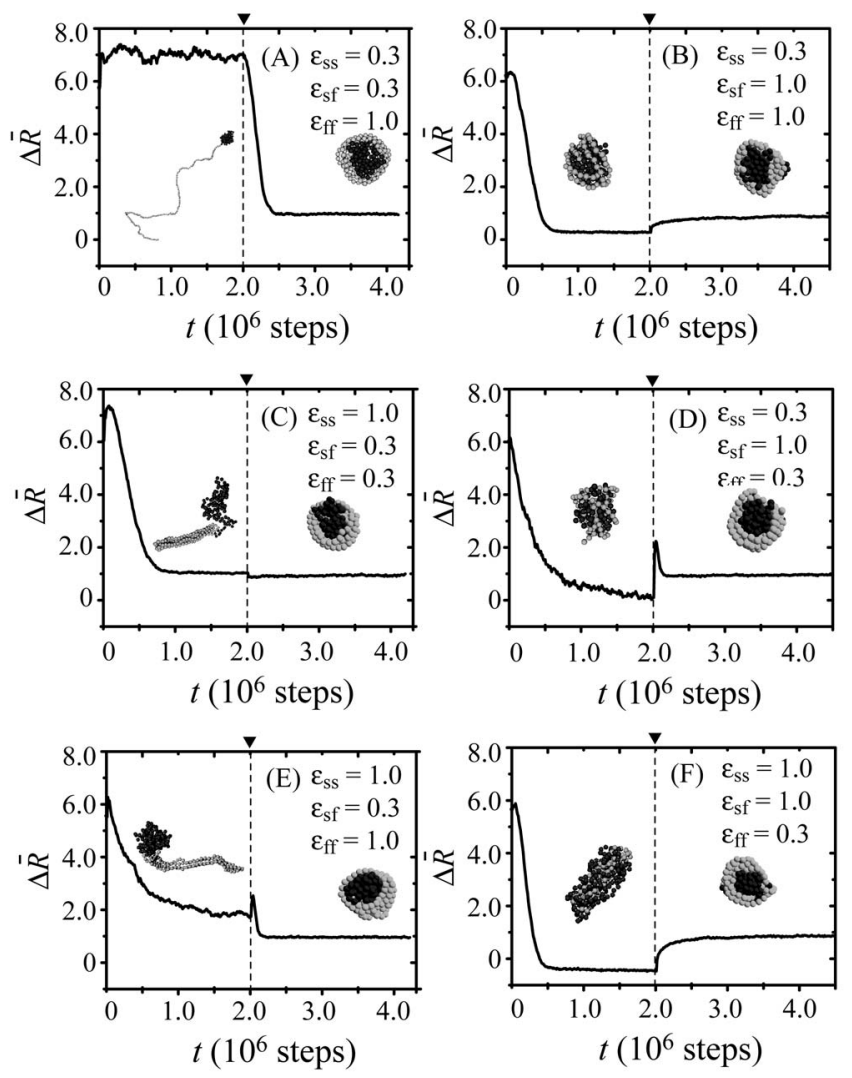

FIG. 3. Pathways of the folding kinetics of a rod-coil copolymer. The effective sizes of the flexible $(\alpha=f)$ and a semiflexible $(\alpha=s)$ blocks, $\bar{R}_{\alpha}$ are defined as $\bar{R}_{\alpha}=\int r \rho_{\alpha}(r) d r / \int \rho_{\alpha}(r) d r$, where $\rho_{\alpha}$ is number density of each block in the radial direction. Here, $r$ represents the distance from the center of mass. The value of $\Delta \bar{R}=\bar{R}_{s}-\bar{R}_{f}$, which represents the extent to which the flexible block is located outside the semiflexible block, is plotted for the various intermediate states. The systems are quenched into six intermediate conditions at $t=0$, and then into the final condition at $t=2 \times 10^{6}$ as indicated by the dashed line. Representations of the initial, intermediate, and final configurations are shown in the figure. The trajectories are averaged over 100 runs.

we consider two-step procedures for changing the parameters, $\left\{\boldsymbol{\epsilon}_{s s}, \boldsymbol{\epsilon}_{s f}, \boldsymbol{\epsilon}_{f f}\right\}$ from $\{0.30,0.30,0.30\}$ to $\{1.0,1.0,1.0\}$. There are six possible intermediate states, as shown in Fig. 3, which have various intermediate conformations depending on the procedures used. Several of the intermediate states show cylindrical shapes in their semiflexible parts. Therefore these structures could be considered to be metastable states because the core-shell shape mentioned earlier contains a toroidal conformation. However, this is not the case. Interestingly, the final structure does not depend on the procedures used. This indicates that our core-shell structure is also independent of the order in which the procedure takes place. This point is clearly demonstrated in the case of $\left\{\epsilon_{s s}, \epsilon_{s f}, \epsilon_{f f}\right\}=\{1.0,1.0,0.30\}$ [Fig. 3(f)] where $\Delta \bar{R}=\bar{R}_{s}-\bar{R}_{f}$ changes negative to positive. Under these conditions, the intermediate state exhibits a core-shell structure in which the core is the semiflexible block and the shell is the flexible block. As we showed, in the final core-shell structure [Fig. 1(II)] the core is the flexible block and the shell is semiflexible block; therefore, the intermediate state shown in Fig. 3(f) has an inside-out composition. Nevertheless, the 


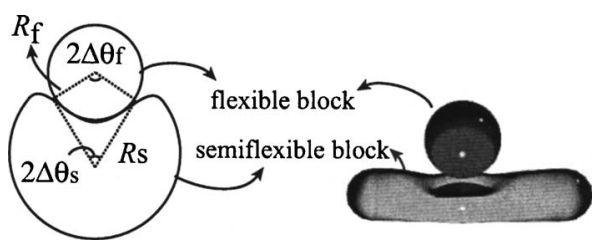

FIG. 4. Deformation of a cylinder resulting from interaction with a globule composed of a flexible block. The left-hand figure shows a cross section of the complex consisting of a spherical globule and a cylinder.

core-shell shown in Fig. 1(II) is always obtained as the final structure.

Why is the core-shell conformation independent of the pathway? First, we should stress that a semiflexible polymer has several metastable states, including a toroid and a cylinder. As shown in Fig. 1(d), the conformation of a semiflexible homopolymer depends on the pathway of the transition. The formation of a cylindrical shape at an early stage of the kinetics leads to a cylinder at the final state. The free energy barrier between the states is sufficiently high that the transition between them does not occur over a practical time scale. However, the addition of a flexible block removes the metastable states. Hereafter, we discuss the reasons for pathway independence. To see the stability of the cylindrical conformation, it is reasonable to assume that the size of the cylinder is of the order of the persistence length, because cylinders obtained experimentally and in simulations are around this size. In this case, the conformational fluctuation is too small to undergo a transition to a toroidal shape when two ends of a cylinder meet. The only pathway from a cylinder to a toroid is to make a hole at the center of a cylinder. We consider a complex of a globule composed of a flexible block and a cylinder composed of a semiflexible block. When the globule is slightly embedded in the cylinder (Fig. 4), the free energy of the system due to the deformation, compared with the reference state, is

$$
\Delta F=F_{\text {int }}+F_{\text {sur }}+F_{\text {bend }} .
$$

The reference state is chosen as the conformation in which the globule and the cylinder are separated, without any interactions. $F_{\text {int }}, F_{\text {sur }}$, and $F_{\text {bend }}$ represent the attractive interaction between the flexible and semiflexible blocks, and the increase in surface energy and the bending energy due to deformation. The energy represented by the first term contributes to deformation, while those of the second and third terms prevent it. Since the attractive interaction is short range, $F_{\text {int }}$ is proportional to the contact area. This energy per unit area can be represented by $-\epsilon_{s f} / a^{2}$, and thus, the free energy is written as

$$
F_{\mathrm{int}} \simeq-\frac{4 \pi \epsilon_{s f} R_{f}^{2}}{a^{2}}\left(\Delta \theta_{f}\right)^{2}
$$

where $\Delta \theta_{f}$ is the angle characterizing the deformation (Fig. 4). The bending free energy at the lowest order in $\Delta \theta_{f}$ is proportional to square of the curvature, which is approximately $\Delta \theta_{f} / L$, and thus

$$
F_{\text {bend }} \simeq \frac{\kappa_{s}}{6 L}\left(\frac{R_{f}}{a}\right)^{2}\left(\Delta \theta_{f}\right)^{4}
$$

where $L$ is the length of the longer axis of the cylinder. With volume conservation and the condition $R_{f} \sin \theta_{f}=R_{s} \sin \theta_{s}$, we find that the surface free energy is proportional to $\left(\Delta \theta_{f}\right)^{4}$,

$$
F_{\text {sur }} \simeq \frac{\pi}{4}\left(\Delta \theta_{f}\right)^{4}
$$

The fact that $F_{\text {int }}$ is dominant for small $\Delta \theta_{f}$ indicates instability in the cylindrical conformation and an increase in contact area. We thus obtain the core-shell conformation as the equilibrium state of the global free energy minimum.

\section{SUMMARY AND REMARKS}

We report novel nanostructures made from single rodcoil block copolymers. The core-shell structure is found to be the equilibrium state. This structure is obtained due to incommensuration of the flexible and semiflexible blocks: The segments in semiflexible blocks tend to align in folded states, while those in flexible blocks tend to be disordered. This state is the counterpart of the coexistence states found in rod-coil copolymer blends. ${ }^{9,18}$

The kinetics of the block copolymers are markedly different from those of semiflexible homopolymers: in a block copolymer, the flexible blocks tend to collapse quickly, which means that the semiflexible blocks can collapse without a nucleation process by making use of the globular collapsed parts of the flexible blocks. On the other hand, semiflexible homopolymers require a long time to enter the folded state because the time required for nucleation appears as a lag time before the collapse. We also discussed the robustness of the core-shell structure: formation of the coreshell structure does not depend on intermediate structures. This was discussed in terms of the instability of cylindrical shapes.

Although our simple model does not specify real macromolecules to which it can be applied, inhomogeneity of elasticity is realized, for example, with binding ligands, ${ }^{19}$ and in mixtures of a helix and a coil ${ }^{20}$ where the helix is relatively stiff. In protein folding, it was proposed that secondary structures are transiently formed at an early stage according to their local preference, independently of native structures. ${ }^{21-23}$ This leads to inhomogeneity of semiflexibility along a chain. Thus, we expect that general features of kinetics at a later stage of proteins in this class are to be described with a mesoscopic model of inhomogeneous bending rigidity. In fact, our polymer has the unique folded structure regardless of difference in pathways. Mesoscopic models for protein folding are still developing. It was proposed that correlation in sequences of hydrophobicity is relevant to the core-shell structure and, in fact, they reproduced the structure with designed sequences. ${ }^{24}$ Our approach focuses instead on semiflexibility along a chain, and we expect that inhomogeneity in semiflexibility plays a key role for transition kinetics in biomacromolecules. 


\section{ACKNOWLEDGMENTS}

The authors thank A. Zinchenko and A. Halperin for reading the manuscript. This work was supported by Japan Society for the Promotion of Science (JSPS) under a Grantin-Aid for Creative Scientific Research (Project No. 18GS0421). One of the authors (N.Y.) would like to acknowledge a fellowship No. 7662 from JSPS. Numerical computation in this work was in part carried out at the Yukawa Institute Computer Facility.

${ }^{1}$ J. Rodrígues-Hernández, F. Chécot, Y. Gnanou, and S. Lecommandoux, Prog. Polym. Sci. 30, 691 (2005).

${ }^{2}$ I. W. Hamley, Nanotechnology 14, R39 (2003).

${ }^{3}$ P. Munk, K. Procházka, Z. Tuzar, and S. E. Webber, CHEMTECH 28, 20 (1998).

${ }^{4}$ I. M. Lifshitz, A. Y. Grosberg, and A. R. Khokhlov, Rev. Mod. Phys. 50, 683 (1978).

${ }^{5}$ T. Sakaue and K. Yoshikawa, J. Chem. Phys. 117, 6323 (2002).

${ }^{6}$ J. F. Marko and E. D. Siggia, Macromolecules 28, 8759 (1995).

${ }^{7}$ J. Ubbink and T. Odijk, Europhys. Lett. 33, 353 (1996).

${ }^{8}$ H. Noguchi and K. Yoshikawa, J. Chem. Phys. 109, 5070 (1998).
${ }^{9}$ A. Nakai, T. Shiwaku, H. Hasegawa, and T. Hashimoto, Macromolecules 19, 3008 (1986).

${ }^{10}$ A. Halperin, Europhys. Lett. 10, 549 (1989).

${ }^{11}$ A. Halperin, Macromolecules 23, 2724 (1990).

${ }^{12}$ S. A. Jenekhe and X. L. Chen, Science 279, 1903 (1998).

${ }^{13}$ I. R. Cooke and D. R. M. Williams, Macromolecules 37, 5778 (2004).

${ }^{14}$ S. Kidoaki and K. Yoshikawa, Biophys. J. 71, 932 (1996).

${ }^{15}$ N. Yoshinaga, K. Yoshikawa, and T. Ohta, Eur. Phys. J. E 17, 485 (2005).

${ }^{16}$ A. Buguin, F. Brochard-Wyart, and P. G. de Gennes, C. R. Acad. Sci., Ser. IIb: Mec., Phys., Chim., Astron. 322, 741 (1996).

${ }^{17}$ N. Yoshinaga, Prog. Theor. Phys. Suppl. 161, 397 (2006).

${ }^{18}$ S. Lee, A. G. Oertli, M. A. Gannon, A. J. Liu, D. S. Pearson, H.-W. Schmidt, and G. H. Fredrickson, Macromolecules 27, 3955 (1994).

${ }^{19}$ K. Besteman, S. Hage, N. H. Dekker, and S. G. Lemay, Phys. Rev. Lett. 98, 058103 (2007)

${ }^{20}$ C. Nowak and T. A. Vilgis, Europhys. Lett. 68, 44 (2004).

${ }^{21}$ Z.-j. Qina, D.-m. Hua, L. Shimada, T. Nakagawa, M. Arai, J.-M. Zhou, and H. Kihara, FEBS Lett. 507, 299 (2001).

${ }^{22}$ O. B. Ptitsyn and A. A. Rashin, Biophys. Chem. 3, 1 (1975).

${ }^{23}$ J. Myers and T. Oas, Annu. Rev. Biochem. 71, 783 (2002).

${ }^{24}$ E. N. Govorun, V. A. Ivanov, A. R. Khokhlov, P. G. Khalatur, A. L. Borovinsky, and A. Y. Grosberg, Phys. Rev. E 64, 040903 (2001). 